Venus Borgohain and Vinay Kumar

\title{
Nanotechnology: Roles in diagnosis and treatment of Covid-19
}

ABSTRACT. Nanotechnology has diverse applications in medical research including viral detection. The potential use of nanotechnology to detect SARS-CoV-2, and to treat and prevent Covid-19, is highlighted in this review. For detection, the isolation of total RNA and characterization using room-temperature loop-mediated isothermal amplification (RT-LAMP) looks promising. Preventive measures such as the development and delivery of vaccines can also be effectively carried out using nanotechnology, especially nanoparticles mediating the efficient delivery of vaccines and for delivering siRNA to specific target sites for silencing viral genes. Other aspects of nanotechnology can also be harnessed in the fight against SARS-CoV-2, including nanomaterials to enhance the efficiency of surgical masks and keep surfaces likely to receive viruses sterile.

Keywords: magnetic nanoparticle, SARS-CoV-2

Nanotechnology Perceptions 17 (2021) 134-140

doi: 10.4024/N20BO20M.ntp.17.02 